\title{
Study of symptoms and gene expression in four Pinus species after pinewood nematode infection
}

\author{
Albina R. Franco, Carla Santos, Mariana Roriz, Rui Rodrigues, \\ Marta R. M. Lima and Marta W. Vasconcelos* \\ Escola Superior de Biotecnologia, Universidade Católica Portuguesa, Rua Dr. António \\ Bernardino de Almeida, 4200-072 Porto, Portugal
}

\begin{abstract}
Pine wilt disease, caused by the pinewood nematode Bursaphelenchus xylophilus (Steiner and Buhrer) Nickle, is originating severe infections in pine trees. The disease is detected when external symptoms appear (e.g. needle chlorosis), but trees could remain asymptomatic for long periods and serve as a long-term host. The primary goal of this study was to assess the effect of inoculation with an avirulent isolate of B. xylophilus (C14-5) on different Pinus spp. seedlings ( $P$. sylvestris, $P$. nigra, $P$. pinea and $P$. pinaster). At the same time, seedlings were also inoculated with a virulent strain, HF, in order to compare the phenotypic and genomic results of the two types of inoculations. The effect of inoculation was determined in terms of expression of various Pinus genes potentially involved in the response to the disease.The results suggest that $P$. pinea and $P$. nigra are more resistant to infection by the nematode than $P$. sylvestris and $P$. pinaster. The phenotypic and genetic differences were more marked among $P$. pinea and $P$. pinaster.
\end{abstract}

Keywords: Bursaphelenchus xylophilus; genetic expression; Pinus spp

\section{Introduction}

Recently, pine wilt disease (PWD), caused by the pinewood nematode (PWN) Bursaphelenchus xylophilus (Steiner and Buhrer) Nickle, has become a major threat to the European forests, with an estimated mortality risk of 50\%. In Portugal, PWN was first detected in 1999 (Mota et al., 1999), and in 2008, the entire continental Portugal was demarcated as PWN-infested. Its insect vector is Monochamus galloprovincialis and, once infected, most plants cease resin production and show the symptoms of needle chlorosis. Infection usually becomes a fatal condition in just a few months (Fukuda, 1997). However, it is not known how the

\footnotetext{
*Corresponding author. E-mail: mwvasconcelos@esb.ucp.pt This project was supported by National Forest Authority, Agriculture Ministry and Rural and Fisheries Development.
}

involved genes are regulated in trees with differential disease resistance after attack by nematodes with varying degrees of virulence (Kosaka et al., 2001; Kuroda et al., 2004). Thus, a targeted gene expression approach was taken in order to investigate the infection mechanisms in commercially important pine species, namely P. sylvestris, which is the most threatened species in northern/central Europe, $P$. nigra and $P$. pinaster, that are being affected in the central/southern areas and $P$. pinea that is thought to be resistant to the infection (OEPP/EPPO, 2001). Symptoms of infection with a virulent strain of PWN (HF, isolated from Setúbal, Portugal) and with an avirulent strain (C14-5, described by Takehushi et al., 2006) were also monitored and evaluated.

\section{Materials and methods}

Seeds of $P$. pinaster, P. pinea, P. sylvestris and P. nigra were sterilized, germinated in $1 \%$ water agar (Agar no. 1 , 
Table 1. Development of symptoms in $P$. pinaster (PP), P. pinea (PPi), P. nigra (Pni), P. sylvestris (Psy), before $\left(T_{0}\right)$, and $10\left(T_{10}\right)$ and $20 \mathrm{~d}\left(T_{20}\right)$ after inoculation with sterile water $\left(\mathrm{H}_{2} \mathrm{O}\right)$, avirulent strain $(\mathrm{A})$ and virulent strain (HF)

\begin{tabular}{|c|c|c|c|}
\hline \multirow[b]{2}{*}{ Treatment conditions } & \multicolumn{3}{|c|}{$\begin{array}{l}\text { Incubation } \\
\text { time }(\mathrm{d})\end{array}$} \\
\hline & $T_{0}$ & $T_{10}$ & $T_{20}$ \\
\hline $\mathrm{PP} \mathrm{H}_{2} \mathrm{O}$ & I & I & II \\
\hline $\mathrm{PP} A$ & I & II & III \\
\hline PP HF & I & III & IV \\
\hline PPi $\mathrm{H}_{2} \mathrm{O}$ & I & I & I \\
\hline PPi A & I & II & II \\
\hline PPi HF & I & l & II \\
\hline Pni $\mathrm{H}_{2} \mathrm{O}$ & I & I & I \\
\hline Pni A & I & II & II \\
\hline Pni HF & I & II & II \\
\hline Psy $\mathrm{H}_{2} \mathrm{O}$ & I & $\mathrm{I}$ & II \\
\hline Psy A & I & II & II \\
\hline Psy HF & I & II & III \\
\hline
\end{tabular}

I, Healthy plant; II, partial needle discolouration; III, partial needle discolouration, necrosis and reduction in the resin production; IV, total discolouration, necrosis and seedling death.

Lab M) and incubated for 2 weeks at $25^{\circ} \mathrm{C}$, with a photoperiod of $8 \mathrm{~h}$ light $-16 \mathrm{~h}$ dark. Once germinated, seedlings were individually incubated vertically for 4 months under the same conditions described earlier and supplied with $10 \mathrm{ml}$ of nutritive solution Murashige and Skoog basal medium (Sigma).

B. xylophilus strains were grown on barley seeds with Botrytis cinerea at $26^{\circ} \mathrm{C}$, in the dark, and extracted using the Baermann funnel technique. A total of 20 seedlings of each pine species were inoculated with 500 avirulent or virulent nematodes in a $100 \mu \mathrm{l}$ sterile water suspension (Asai and Futai, 2002).

To evaluate the genetic expression, samples were taken at 0, 10 and $20 \mathrm{~d}$ after inoculation and stored at $-80^{\circ} \mathrm{C}$. Total RNA was extracted according to Le Provost et al. (2007) and purified with Turbo DNA-free kit (Applied Biosystems, Foster city, CA, USA), according to the manufacturer's instructions. Gene expression was determined using $100 \mathrm{ng}$ of RNA, with the conditions and program presented in Supplementary Table S1 (available online only at http://journals.cambridge.org), using an MJ Mini Gradient Thermal Cycler (Bio-Rad Laboratories, PA, USA). The gene $18 S$ was used as internal control.

Scanning electron microscope was used to examine the morphology of $P$. pinea and $P$. pinaster stems. Thin, manual cuts were made with a scalpel. Each sample was attached to a support with double-sided duct tape and placed in a desiccator until the samples were dehydrated. Samples were analysed following the user manual of SEM JSM5600LV, operating at $20 \mathrm{kV}$.

\section{Results and discussion}

Symptoms of disease were only detected $10 \mathrm{~d}$ after inoculation. $P$. pinea and $P$. nigra seem less susceptible to the infection since their symptoms did not develop beyond stage II; $P$. pinaster appears to be the most susceptible species, as some seedlings died $20 \mathrm{~d}$ after the inoculation. Also, inoculation with the avirulent nematode resulted in seedlings with some degree of needle discolouration. (Table 1) These differences of infection may be due to the blocking of the vascular system with resin produced by cells from radial parenchyma, which are damaged by the nematode, resulting in needle chlorosis and plant death some time after infection (Jones et al., 2008).

Anatomical differences among genotypes might be on the basis of differential resistance to PWN, therefore $P$. pinaster and $P$. pinea were examined with scanning electron microscope (SEM). Visual inspection of the resulting photographs (Fig. 1) indicates that the round shape of the stem is better maintained in $P$. pinea than in $P$. pinaster after manual cross-sectioning. This may be due to higher lignin content in $P$. pinea and may be related to increased resistance to PWD in this species. Lignin has a recognized role in plant defence, and constitutive lignin has already been related with
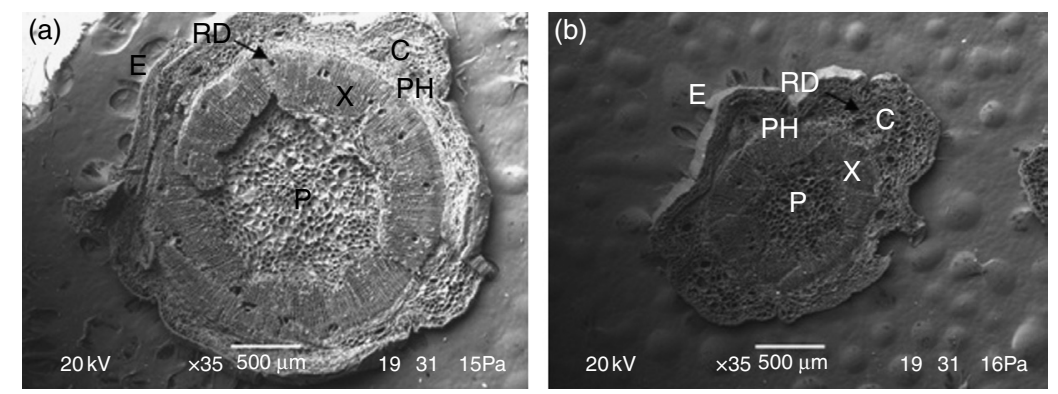

Fig. 1. SEM imaging of $P$. Pinea (a) and $P$. pinaster (b) stem cross-sections showing morphological differences between the two species. E, epidermis; C, cortex; RD, resin duct; $\mathrm{PH}$, phloem; $\mathrm{X}$, xylem; $\mathrm{P}$, pith. 
defensive mechanisms against nematodes in other species (Fogain and Gowen, 1996). Experiments regarding lignin quantification are ongoing. Furthermore, the diameter in cortical resin ducts seems larger in $P$. pinaster stem. This may contribute to the susceptibility of this genotype to PWD, since PWNs progress inside the plant through resin ducts (Fukuda, 1997). Increased number and diameter of resin ducts have already been associated with PWD susceptibility (Kawaguchi, 2006).

Biotic and abiotic factors stimulate the plant's defence response, diminishing the negative impacts of the pathogenic attack. The genes of interest tested in this work were found to be associated to osmotic stress, oxireductive processes and cell death, among others, which are important in the defence response of $P$. densiflora (Japanese Red Pine) against the nematode (Shin et al., 2009).

Pathogenesis-related proteins 4 expression was detected in all treatments (Supplementary Figs. S1-S4, available online only at http://journals.cambridge.org). PR proteins are induced as response to pathogen attacks (Osmond et al., 2001) and can be factors of hypersensitive response to nematode infection (Meins and Ahl, 1989; Shin et al., 2009). ATTRX1, a protective gene against oxidative stress, was also expressed in all treatments and pine tree species (Supplementary Figs. S1-S4, available online only at http://journals.cambridge.org), suggesting that different types of defences may be activated. There is a reported relationship between metallothionein being expressed in the presence of intensive oxidative stress (Mir et al., 2004).

Ethylene is an important component of conifer response against pathogens (Miller et al., 2005), hence it can induce cell defence. In P. pinaster (Supplementary Fig. S1, available online only at http://journals.cambridge.org), the expression of MAT2/SAM2 (ethylene production) was only detected at the end of $20 \mathrm{~d}$, which in the case of virulent B. xylophilus treatment corresponded to plant death; in $P$. pinea (Supplementary Fig. S2, available online only at http://journals.cambridge.org), the inoculations with both avirulent and virulent nematodes originated the same type of response as water-inoculated plants (control). On the other hand, in seedlings of P. nigra (Supplementary Fig. S3, available online only at http://journals.cambridge.org), MAT2/SAM2 and SHEPERD (water reduction) gene expression was only verified in nematode-inoculated plants. This can be explained by the increasing release of volatile compound production, which alters water transportation (Jones et al., 2008). Finally, P. sylvestris seedlings (Supplementary Fig. S4, available online only at http://journals.cambridge.org) demonstrated that virulent strains of B. xylophilus did not cause any MAT2/SAM2 gene expression.

It must be noted that some of the primers used (Supplementary Table S2, available online only at http://journals.cambridge.org) were not specifically designed for the species in question. Thus, it could explain the absence of genes that normally would be expressed. Also, plant mechanisms vary during the time of a day, so the time when the sampling was made may also have influenced the results.

This is the first report of inoculations with virulent and avirulent B. xylophilus strains in various pine species, and though infection mechanisms of both PWN were not clear, this study suggests that inoculation with virulent nematode can trigger a phased systemic response that differs from the avirulent strain. However, it is necessary to identify other factors that may be responsible for the plant defence when it is attacked by the different pathogens.

\section{Acknowledgements}

The authors would like to thank the help of Dr. Manuel Mota (Universidade de Évora, Portugal) for providing the HF nematode strain, and Dr. Hajime Kosaka (Kyushu Research Center Forestry and Forest Products Research Institute, Kumamoto, Japan) and Dr. Mitsutera Aikida (Forestry and Forest Products Research Institute, Tsukuba, Japan) for providing the C14-5 nematode strain.

\section{References}

Asai E and Futai K (2002) Promotion of the population growth of pinewood nematode in 4-month-old. Journal of Forestry Research 7: 113-116.

OEPP/EPPO (2001) Bursaphelenchus xylophilus. $P M 7 / 4$ (1) OEPP/EPPO Bulletin 31: 61-69.

Fogain R and Gowen SR (1996) Investigations on possible mechanisms of resistance to nematodes in Musa. Euphytica 92: $375-381$.

Fukuda K (1997) Physiological process of the symptom development and resistance mechanism in pine wilt disease. Journal of Forest Research 2: 171-181.

Jones J, Moens M, Mota M, Li H and Kikuchi I (2008) Bursaphelenchus xylophilus: opportunities in comparative genomics and molecular host-parasite interactions. Molecular Plant Pathology 9: 357-368.

Kawaguchi E (2006) Relationship between the anatomical characteristics of cortical resin canals and migration of Bursaphelenchus xylophilus in stem cuttings of Pinus thunbergii seedlings. Journal of the Japanese Forest Society 88: $240-244$.

Kosaka H, Aikawa T, Ogura N, Tabata K and Kiyohara T (2001) Pine wilt disease caused by the pine wood nematode: the induced resistance of pine trees by the avirulent isolates of nematode. European Journal of Plant Pathology 107: $667-675$.

Kuroda K (2004) Inhibiting factors of symptom development in several Japanese red pine (Pinus densiflora) families 
selected as resistant to one wilt. Journal of Forestry Research 9: 217-224.

Le Provost G, Herrera R, Paiva J, Chaumeil P, Salin FF and Plomion C (2007) A micromethod for high throughput RNA extraction in forest trees. Biological Research 40: 291-297.

Meins F and Ahl F (1989) Induction of chitinase and [beta]-1, 3-glucanase in tobacoo plants infected with Pseudomonas tabaci and Phytophythora parasitica var. nicotianae. Plant Physiology 61: 155-161.

Miller B, Madilao L, Ralph S and Bohlmann J (2005) Insectinduced conifer defense, White pine weevil and methyl jasmonate induce traumatic resinosis, de novo formed volatile emissions, and accumulation of terpenoid synthase and putative octadecanoid pathway transcripts in Sitka spruce. Plant Physiology 137: 369-382.

Mir G, Demenech G, Huguet G, Guo W, Goldsbrough P, Atrian S, Molinas M (2004) A plant type 2 metallothionein (MT) from cork tissue responds to oxidative stress. Journal of Experimental Botany 55: 2483-2493.

Mota MM, Braasch H, Bravo MA, Penas AC, Burgermeister W, Metge K and Sousa E (1999) First report of Bursaphelenchus xylophilus in Portugal and in Europe. Nematology 1: $727-734$.

Osmond R, Hrmova J, Fontaine F, Imberty A and Fincher G (2001) Binding interactions between barley thaumatin-like proteins and (1,3)- $\beta$-d-glucans. European Journal of Biochemistry 268: 4190-4199.

Shin H, Lee H, Woo K-S, Noh E-W, Koo Y-B and Lee K-J (2009) Identification of genes upregualted by pinewood nematode inoculation in Japanese red pine. Tree Physiology 29: 411-421.

Takehushi Y, Kanzaki N and Futai K (2006) How different is induced resistance against the pine wood nematode, Bursaphelenchus xylophilus, by two avirulent microbes? Nematology 8: 435-442. 